REVISTA ANDALUZA DE ANTROPOLOGÍA.

NÚMERO12:PATRIMONIOINMATERIAL:REDUCCIONISMOS, CONFLICTOSE INSTRUMENTALIZACIONES. INTANGIBLE CULTURAL HERITAGE: REDUCTIONISMS, CONFLICTS AND INSTUMENTALIZATIONS.

MARZO DE 2017

ISSN 2174-6796

[pp. 48-69]

http://dx.doi.org/10.12795/RAA.2017.12.03

Recibido: 03/11/2016

Aceptado: 23/01/2017

\title{
LOS VERBOS DE LA PARTICIPACIÓN SOCIAL Y SUS CONJUGACIONES: CONTRADICCIONES DE UN PATRIMONIO “DEMOCRATIZADOR"1
}

\section{THE VERBS OF SOCIAL PARTICIPATION AND ITS CONJUGATIONS: CONTRADICTIONS OF A “DEMOCRATIZING" HERITAGE}

\author{
Victoria Quintero Morón \\ Universidad Pablo de Olavide \\ Cristina Sánchez Carretero \\ Instituto de Ciencias del Patrimonio (Incipit), CSIC
}

\section{Resumen.}

El objetivo de este artículo ${ }^{1}$ es reflexionar sobre algunas de las propuestas y paradojas de las innovaciones que introduce la Convención para la Salvaguardia del Patrimonio Cultural Inmaterial de 2003 y su relación con la participación social. Para ello se introduce el debate que en los últimos años se ha producido en antropología, museología y disciplinas afines centrándonos en dos aspectos: las paradojas que implica el uso de la expresión "comunidades, grupos y en algunos casos individuos" en la Convención de 2003 y en las implicaciones del giro participativo para la gestión patrimonial y su pretendido efecto democratizador. Se presta especial atención a la distancia entre los diferentes infinitivos vinculados a la participación (los "verbos" de la participación) y la forma en la que se ponen en acción (las "conjugaciones").

1 Este artículo se enmarca dentro del proyecto de I+D del Plan Nacional, ParticiPat, "Patrimonio y participación social: propuesta metodológica y revisión crítica” (HAR2014-54869-R) y del proyecto NEARCH financiado por el programa CULTURA de la Comisión Europea. 


\title{
Palabras claves.
}

Patrimonio inmaterial; Participación social; Régimen patrimonial; Convención de 2003

\begin{abstract}
.
The goal of this article is to analyze some of the proposals and paradoxes of the 2003 Intangible Heritage Convention in relation to social participation. To do so, we introduce the debates that have taken place over the last decades in anthropology, museology and nearby disciplines. The article has a twofold focus: the paradoxes embedded in the expression "communities, groups and, if applicable, individuals" of the 2003 Convention;and the outcomes of the participatory turn in heritage management and its alleged democratizing effect. Special attention is dedicated to the different infinitives linked to participation (the "verbs" of participation) and the different ways in which participation is enacted (the "conjugations").
\end{abstract}

\section{Key Words.}

Intangible heritage; Participation; Heritage Regime; 2003 ICH Convention

\section{INTRODUCCIÓN}

Recientemente, en un coloquio en torno a los Patios de Córdoba y el Patrimonio Inmaterial $^{2}$, una de las participantes se preguntaba si estábamos pensando en los deseos y aspiraciones reales de las personas que habitaban los patios; cuestionaba quiénes querrían seguir viviendo en casas muy envejecidas, con mucha humedad, sin aire acondicionado, muy costosas de mantener tanto en recursos económicos como en trabajo... Si no era lógico pensar -seguía argumentando- que los jóvenes se quisieran marchar y no diesen continuidad a los "saberes" y modos de vida de sus mayores, puesto que no tenía sentido paralizar el tiempo y puesto que las cosas han cambiado para mejor en cuanto a la habitabilidad en las viviendas actuales. En sencillas y sabias palabras, esta vecina planteaba hasta qué punto no se estaban idealizando las tradiciones; si no se estaba construyendo un escenario para que otros pudieran ver una realidad que ya no tenía razón de ser y de qué modo se estaban teniendo en cuenta las vivencias cotidianas y los problemas reales de ser un habitante de unos espacios considerados parte del "Patrimonio Oral e Intangible de la Humanidad" según la Convención para la Salvaguarda del Patrimonio Cultural Inmaterial de 2003 de la UNESCO³ (en adelante Convención de 2003).

2. Programa de "Diálogos con la Cultura" organizado por la Facultad de Filosofía y Letras de la Universidad de Córdoba, coordinado por el profesor José María Manjavacas. La sesión se titulaba "Patios y Gentes. Patrimonio Inmaterial y Compromiso Ciudadano" y se celebró el 14 de octubre de 2016. 
En pocas palabras esta vecina centró la problemática de forma práctica en el habitar, en los cuidados, en los deseos y expectativas de la gente que habita esos espacios, así como la sostenibilidad del modo de vida que hace posible seguir manteniéndolos y los problemas de la gentrificación. Esta intervención tan directa y conectada con la realidad del día a día de los llamados "portadores" o "depositarios" del patrimonio, nos sirve de marco para reflexionar sobre algunas de las propuestas y contradicciones de las innovaciones que introduce la Convención de 2003 y su relación con la participación social.

Dentro de la antropología del patrimonio se llevan décadas insistiendo en la necesidad de entender el patrimonio de forma holística y los problemas que trae consigo la compartimentalización en categorías dicotómicas que reproducen un sistema de pensamiento basado en la propiedad de exclusión: patrimonio material/inmaterial; patrimonio cultural/ natural; o patrimonio de élites o artístico frente a patrimonio popular o folklore. También existen otras dicotomías no estandarizadas en etiquetas pero no por ello menos reproducidas -ya que se han naturalizado en la gestión patrimonial- como el patrimonio para las poblaciones locales/patrimonio para los turistas; valor de uso/valor de cambio; o patrimonio del norte/del sur. Si bien la superación de estas dicotomías se ha dado en un plano analítico, el llamado "régimen patrimonial" (Hafstein, 2012; Bendix, Eggert y Peselmann, 2012) las reproduce en sus diferentes niveles.

En este artículo queremos señalar la reproducción de dicotomías vinculadas a la categoría "patrimonio inmaterial" y las contradicciones que esto implica; centrándonos especialmente en las contradicciones que la implementación de la participación está generando. Para ello, nos parece importante pensar el patrimonio como régimen, vinculándolo a la idea de "regímenes de verdad" de Foucault, como sistema de procedimientos ordenados para la producción, regulación, circulación y funcionamiento de afirmaciones que no se cuestionan (Foucault 1977). La idea de "régimen patrimonial" ha sido usada por Hafstein (2012) y Bendix, Eggert y Peselmann (2012) en el mismo sentido en el que Alonso González usa el término "máquina patrimonial" (2013) o Jeudy la expresión "la machinerie patrimoniale" (2001).

La perspectiva de entender el engranaje sobre el que se articulan las estructuras patrimoniales y el "discurso patrimonial autorizado" (Smith 2006) se ha vinculado en el mundo anglófono -y francófono canadiense- a los llamados "estudios críticos de patrimonio", que se han desarrollado de la mano de la Asociación de Estudios Críticos de Patrimonio. Como indican en el manifiesto inaugural de esta asociación, uno de sus objetivos es "invitar a que participen activamente la gente y las comunidades que hasta el

3. La Fiesta de los Patios de Córdoba forma parte del listado de UNESCO desde 2012. Un resumen de los valores y la documentación sobre esta declaración puede consultarse en la página web de UNESCO http://www.unesco.org/culture/ich/es/RL/la-fiesta-de-los-patios-de-cordoba-00846 
momento han sido marginalizadas de la creación y gestión del patrimonio"4. Un objetivo común con el imperativo de la participación que analizaremos aquí.

\section{UN PATRIMONIO “DEMOCRATIZADOR": DESAFÍOS Y PARADOJAS DEL PATRIMONIO INMATERIAL}

Si retomamos el coloquio mencionado al principio del texto sobre los habitantes de los Patios de Córdoba, en las palabras de esta señora se alude por una parte, a la idea de la dinámica cultural -o como se afirma por parte de la UNESCO, al principio de que el Patrimonio Cultural Inmaterial (PCI) es un patrimonio vivo- y por otra parte, al protagonismo que se otorga a las "comunidades, grupos y en algunos casos individuos" en el“nuevo paradigma participativo" del patrimonio (Adell et al., 2015). En los siguientes párrafos vamos a tratar sintéticamente estas nociones en el marco de la Convención, en cómo influye y se desborda en distintas escalas de los regímenes patrimoniales y en las paradojas y desafíos a los que nos enfrenta.

A pesar de las apariencias, la patrimonialización de ciertas prácticas culturales -eso que hoy damos en llamar patrimonio inmaterial- no puede considerarse una acción muy novedosa, puesto que existen precedentes directos tanto en acciones museísticas -museos al aire libre, ecomuseos, museos de comunidad, vecinales...- (Quintero, 2005; Santamarina, 2013), como en el tratamiento del folklore (Hymes, 2000 [1975]) , del llamado folklore público (Baron y Spitzer, [1992] 2007] y también en las legislaciones patrimoniales estatales en referencia a las actividades, saberes y prácticas consideradas parte del "patrimonio etnológico"(Rioja, 1996; Agudo, 1999; Pérez Galán, 2011). La noción fue transformándose: durante los treinta años que preceden a la publicación de la Convención de 2003 hubo trabajo de expertos y un debate internacional sobre el tratamiento de este tipo de patrimonio (Kurin, 2004) ${ }^{6}$ y una evolución del concepto por ejemplo de la Convención de 1989 a la de 2003-. Pero, en paralelo a ese proceso en UNESCO, en los entornos de los distintos Estados-Nación la expansión del patrimonio etnológico -o etnográfico- era una pauta en la que cada vez más se patrimonializaban también prácticas culturales. De hecho, ese crecimiento del patrimonio etnológico en las últimas décadas del siglo XX visibilizaba un conjunto de transformaciones de carácter

4. La asociación se formó a raíz de un manifiesto leído en 2012 en el primer congreso de la asociación en Goteborg, Suecia. Véase la web de la asociación http://www.criticalheritagestudies.org/

5. Dell Hymes, ya en 1975 introdujo el concepto de "procesos de tradicionalización" para superar la visión inmovilista de los estudios sobre el folklore (Hymes, 2000).

6. Como explica Koïchiro Matsuura, que fue director general de la UNESCO entre 1999 y 2009, se trabajó para introducir la categoría de PCI con un fin compensatorio norte-sur, para corregir el desequilibrio entre los lugares declarados patrimonio de la humanidad en ambos hemisferios (Matsuura, 2004:4). 
económico y político que hunden sus raíces en la expansión neoliberal y del proceso de globalización ${ }^{7}$. En definitiva, la noción de PCI no es novedosa, véanse por ejemplo, las similitudes de la definición de PCI de la Convención de 2003 de la UNESCO con la definición de folklore de 1846 de William Thoms (Sánchez-Carretero, 2005: 149); aunque sí que lo son las formas en que se define como patrimonio (Kirshenblatt-Gimblett, 2004; Arizpe, 2004; Quintero, 2005; Pérez Galán, 2008; Bortolotto, 2011; Villaseñor y Zolla, 2012; Santamarina, 2013) y una serie de nuevas relaciones y protagonismos entre los actantes de la patrimonialización (Tauschek, 2015; Adell et al., 2015) ${ }^{8}$.

La presentación de prácticas, expresiones, saberes, rituales y performances en este "nuevo envoltorio" del PCI (Santamarina, 2013) ha tenido bastante éxito ${ }^{9}$ sigue la estela de la expansión patrimonial de las últimas décadas (Ariño, 2002) ${ }^{10}$. Esta capacidad de difusión y la aceptación con que se ha acogido el PCI se relaciona con un giro en el discurso de la UNESCO que va del exotismo del pasado a una pretensión de ensalzar

7. Desde el punto de vista político, el auge de estos patrimonios visibilizaba diversos procesos de etnización y reconstrucción nacional, tanto para la construcción de nacionalismos periféricos y regionalismos (Albert, 2003), como en el apuntalamiento de identidades nacionales poscoloniales uniendo tradiciones y colectivos étnicos diferenciados bajo la idea de un mismo Estado-Nación (Collomb, 1999; Segalen, 2003). Desde el punto de vista más ligado a los procesos económicos globales, la expansión de "nuevos patrimonios" -como el denominado etnológico- está claramente conectado con el desarrollo y las nuevas necesidades de la industria turística (Alsayyad, 1999; Santana, 2002; Prats, 2003) y con la reestructuración de ciertas áreas -consideradas periféricas respecto a otros procesos productivos- que se pretenden reorientar hacia los servicios y el turismo (Aguilar y Amaya, 2007; Pazos Cárdenas, 2016).

8. Tauschek (2015) propone aplicar la teoría del actor-red considerando que las distintas formas de patrimonialización deben ser analizadas a través de las relaciones e interacciones del conjunto de actantes humanos (depositarios heterogéneos, empresarios, expertos, políticos, turistas, etc.) y nohumanos (normas, procedimientos, referencias...). Afirma que los actores no-humanos, como las infraestructuras burocráticas, los protocolos, guidelines y los textos legales "son muy importantes en la emergencia y distribución global de los regímenes patrimoniales porque garantizan la interpretación común de conceptos, definiciones y parámetros legales y definen estrictamente los procesos" (2015: 300, la traducción es nuestra).

9. Un éxito que se refleja en los 170 países que han firmado la Convención de 2003 hasta ahora (datos de UNESCO a fecha 10/6/2016), pero, lo que nos parece aún más importante, por cómo este modelo del PCI está afectando a los regímenes patrimoniales estatales (Bendix et al. 2012); y cómo está remodelando las relaciones entre expertos y ciudadanos (Adell et al. 2015) -aunque esto último todavía tiene más efecto en las legislaciones y los protocolos que en las prácticas de gestión-, Bruman llega a afirmar que la Convención del Patrimonio Mundial es en la actualidad "la arena global con más influencia en la conservación" (2015:274, la traducción es nuestra).

10. Un éxito que se refleja en los 170 países que han firmado la Convención de 2003 hasta ahora (datos de UNESCO a fecha 10/6/2016), pero, lo que nos parece aún más importante, por cómo este modelo del PCI está afectando a los regímenes patrimoniales estatales (Bendix et al. 2012); y cómo está remodelando las relaciones entre expertos y ciudadanos (Adell et al. 2015) -aunque esto último todavía tiene más efecto en las legislaciones y los protocolos que en las prácticas de gestión-, Bruman llega a afirmar que la Convención del Patrimonio Mundial es en la actualidad "la arena global con más influencia en la conservación" (2015:274, la traducción es nuestra). 
y acoger la diversidad cultural, dando cabida a producciones y saberes de pueblos y grupos claramente infrarrepresentados en las listas declaratorias del patrimonio mundial (Kirshenblatt-Gimblett, 2004; Matsuura, 2004; Santamarina, 2013). Tampoco se puede obviar que la "fama" obtenida por estas fórmulas de patrimonialización se relaciona con las siempre crecientes demandas de la industria turística en busca de nuevos mercados y productos. Son diversos los autores que señalan, críticamente, el éxito en mercadotecnia que supone ser parte de las Listas de Patrimonio de la Humanidad y las implicaciones que ello tiene en las definiciones de los bienes -materiales e inmateriales-, así como las transformaciones que supone en las sociedades receptoras (Villaseñor y Zolla, 2012).

Sin embargo, parte del éxito y expansión de la Convención de 2003 se debe a su propuesta democratizadora que, además de visibilizar a nuevos colectivos, da expresamente el protagonismo de la patrimonialización a las "comunidades, grupos y en algunos casos individuos" pues son sólo ellos los capacitados para "reconocer como parte integrante de su identidad cultural" a "los usos, representaciones, expresiones, conocimientos y técnicas" que constituyen el PCI, tal como se afirma en el art. 2 de la Convención ${ }^{11}$. Es decir, son las "comunidades, grupos y en algunos casos individuos" los que deben identificar, definir y diseñar, entre otros, los inventarios, programas y nominaciones. Esta relevancia de las comunidades y grupos supone un giro radical, en teoría, respecto a las lógicas y prácticas de las Convenciones anteriores de la propia UNESCO que depositaba en los técnicos, expertos y académicos la asignación de un valor supuestamente objetivo y universal a los bienes culturales. En las visiones hegemónicas del patrimonio hasta la aparición de la Convención de 2003, la legitimidad para la selección y la atribución de valores a los objetos patrimoniales -incluso a las prácticas y conocimientos tradicionales o populares- provenía exclusivamente de los expertos. Depositar la legitimidad de la patrimonialización en las comunidades y grupos locales supone una transformación profunda: es lo que ciertos autores denominan como el nuevo "paradigma participativo del patrimonio" (Adell et al., 2015; Sánchez-Carretero y Jiménez-Esquinas, 2016; Cortés Vázquez, Jiménez-Esquinas y Sánchez-Carretero, 2017) inscrito en los actuales modelos de gobernanza neoliberal. Este cambio, al mismo tiempo, es un proceso que continúa la lógica de los últimos 50 años en la transformación del concepto de patrimonio; donde

11. Artículo 2: "Definiciones. A los efectos de la presente Convención, 1. Se entiende por "patrimonio cultural inmaterial" los usos, representaciones, expresiones, conocimientos y técnicas -junto con los instrumentos, objetos, artefactos y espacios culturales que les son inherentes- que las comunidades, los grupos y en algunos casos los individuos reconozcan como parte integrante de su patrimonio cultural. Este patrimonio cultural inmaterial, que se transmite de generación en generación, es recreado constantemente por las comunidades y grupos en función de su entorno, su interacción con la naturaleza y su historia, infundiéndoles un sentimiento de identidad y continuidad y contribuyendo así a promover el respeto de la diversidad cultural y la creatividad humana. A los efectos de la presente Convención, se tendrá en cuenta únicamente el patrimonio cultural inmaterial que sea compatible con los instrumentos internacionales de derechos humanos existentes y con los imperativos de respeto mutuo entre comunidades, grupos e individuos y de desarrollo sostenible." Convención para la Salv 
se produce un giro del Arte y la Historia a la Cultura, de la universalidad a la identidad y de las obras maestras a la representatividad (Agudo, 1999; Hafstein, 2009; Querol, 2010). La integración de las comunidades y grupos locales en la selección del patrimonio, en la definición de qué valores contiene y representa, en la decisión de cómo transmitirlo, transformarlo y usarlo supone, sin duda, una transformación de las jerarquías habituales de la patrimonialización y también dotar de voz y de visibilización en la "vitrina del patrimonio" a colectivos minorizados, subalternos, poco visibles... Supone también cuestionar la supremacía de las creaciones occidentales basadas en la materialidad, en la obra de grandes arquitectos o artistas, creadas para el consumo prioritario de las élites, para el mantenimiento de su poder, para la reproducción de su distinción (Bourdieu, 1987).

Este protagonismo de comunidades y grupos autóctonos es además necesario para mantener y transmitir un patrimonio que se define como "vivo", dinámico, sujeto a hibridaciones y cambios... y que reposa en los quehaceres, prácticas, sentires y expresiones de sus usuarios: ¿cómo celebrar Carnaval de Cádiz sin sus protagonistas, sus chirigotas, sin los gaditanos?, ¿cómo mantener los saberes en torno a la cal sin los caleros? ¿cómo dar continuidad a una cocina sin los paisajes, los procedimientos, los pequeños trucos, los conocimientos y las formas de compartir de cocineras y comensales?

\section{ENTORNO AL GRUPO “DEPOSITARIO" Y AL CONCEPTO DE “COMUNIDAD, GRUPOS Y EN ALGUNOS CASOS INDIVIDUOS”}

Sin embargo, uno de los principales problemas del giro participativo en el que el protagonismo pasa a los colectivos en este "hacer el patrimonio" se sitúa en cómo se definen esos colectivos. Por ejemplo, ¿quiénes componen el grupo "depositario" o "comunidad" de los saberes y prácticas asociados al Flamenco, a la Dieta Mediterránea, a la Fiesta de los Patios de Córdoba, a la Patum de Berga? En el primer caso, se podría pensar que el flamenco se circunscribe a los artistas, los aficionados, los flamencólogos, los empresarios de flamenco... pero,¿̇ónde quedan los amateurs, o los públicos no expertos? En el caso de la Fiesta de los Patios, ¿son los vecinos de esos patios, es decir, de los patios que participan en el concurso? O, por el contrario, ¿se debería ampliar a los vecinos de Córdoba que hacen posible la fiesta? ¿y a los turistas que acuden a ella? En este sociograma de la Fiesta de los Patios también podrían figurar los políticos locales, técnicos y expertos, así como las instancias político-administrativas autonómicas y estatalesque promueven o se resisten a la patrimonialización,

Estas y muchas otras cuestiones forman parte del presente debate en torno a cómo definir a las "comunidades, grupos y en algunos casos individuos" que protagonizan o forman 
parte de los procesos de patrimonialización. Veamos concretamente estas fracturas y contradicciones de las nociones de comunidad y grupos propuestas en los textos y protocolos patrimoniales recientes.

Especialistas en antropología vienen señalando que el concepto de comunidad que utiliza la Convención de 2003 y otros documentos de UNESCO se caracterizan por definirla de un modo idealizado, normalizado y bien delimitado. Es decir, se produce una esencialización de la comunidad (Arrieta, 2011; Hertz, 2015; Kirshenblatt-Gimblett, 2004; Tauschek, 2015; Villaseñor y Zolla, 2012; Waterton y Smith, 2010), considerándola inherentemente buena, exenta de conflictos, homogénea, sin jerarquías internas, con una sola voz. En realidad, los colectivos o comunidades inmersos en o afectados por procesos de patrimonialización, son heterogéneos en su composición e intereses, tienen diferentes percepciones de la acción o del ritual o del procedimiento y le atribuyen a menudo significados distintos. De hecho, como numerosos autores vienen señalando, la arena patrimonial se define como un espacio de conflicto (García, 1999; Rosas, 1999; Santamarina, 2005; Quintero, 2005; Pérez Galán, 2008; Sánchez-Carretero, 2012a) puesto que pone de relieve las contradicciones y tensiones inherentes a la sociedad desigual y compleja que habitamos. Los procesos de patrimonialización, en tanto que suponen la selección de unos elementos y por tanto excluyen a otros, (Tunbridge y Ashworth, 1996) y en tanto que obligan a una resignificación de esos bienes y a una reducción de su polisemia, son procesos en los que las alianzas, las negociaciones y los conflictos son frecuentes (Sánchez-Carretero y Jiménez-Esquinas, 2016) y donde la heterogeneidad de intereses y de poder se muestran.

De ahí que el giro participativo suponga un desafío y una paradoja ya que desde un principio nos lleva a señalar o delimitar quiénes conforman la comunidad o el grupo en la patrimonialización. A este propósito la antropóloga Ellen Hertz (2015) muestra cómo en las definiciones de "comunidad, grupos y en algunos casos individuos" de los textos internacionales aparecen tres conceptos diferentes de comunidad o colectivo que se usan indistintamente y se mezclan, pero que sin embargo, tienen efectos prácticos muy distantes entre sí. Esta autora, siguiendo a Noyes (2012) distingue entre: un concepto fundacional de comunidad (es un colectivo esencialmente definido, por su vinculación con la tierra y la historia), un concepto relacional (un colectivo que se define por oposición a otros, que son diferentes y antagónicos) y un concepto performativo (un colectivo que se performa, se define y reconstituye en el "hacer patrimonio", en la misma transformación social que "hace" el patrimonio). Los dos primeros conceptos son los que aparecen implícitos en la Convención de 2003, están ligados a comunidades (o grupos) étnica e históricamente definidos y tienen cierto sesgo esencialista; el tercero se opone a estos dos, se concibe como red y no existiría independientemente del patrimonio (Hertz, 2015), es decir, se conforma a través de la patrimonialización. Esta última es lo que en 
la Convención de Faro de 2005 se ha denominado "comunidad patrimonial" y lo que proponen los académicos en el Expert Report on Community Involvement de UNESCO de 2006.

Si seguimos el ejemplo de la Fiesta de los Patios de Córdoba, la definición de "comunidad o grupo" según el primer concepto -la definición fundacional o esencialista de comunidadreferiría prioritariamente a los vecinos de los patios que consuetudinariamente han venido habitando en ellos y presentándose al Concurso, es decir, participando en la fiesta y también son los que han desarrollado los modos de vida ligados a los patios, los depositarios de los saberes en torno a las plantas y los ciclos de la ciudad... Sin embargo, esta delimitación resulta confusa por cuanto que quiénes sean esos habitantes es un concepto flexible que no puede restringirse a una comunidad o grupo primigenio e intocado: dentro de las dinámicas urbanas de los centros históricos en las últimas décadas ha habido una gran transformación, muchos de los inquilinos de los patios de vecinos viven hoy en barriadas de la periferia, los patios comunitarios son hoy escasos y esos modos de vida están casi desaparecidos y nuevos habitantes han venido a sustituir a los antiguos: ¿¿cuál es la "auténtica" comunidad aquí? Además, esta comunidad, ¿incluye sólo a los que participan activamente? ¿no debería incluir a todos los cordobeses que celebran la fiesta? Pero, ¿quiénes conforman ese "todos"? La transformación de las comunidades y los grupos, la aparición de nuevos habitantes, de migrantes, las mezclas y las hibridaciones son constantes en el mundo actual e imposibles de encajar en esta definición esencialista de comunidad.

Sin embargo, si nos vamos a la "comunidad patrimonial" o "concepción performativa" de las comunidades y grupos, el problema no desaparece. Es cierto que esta noción se adapta mejor a la idea de patrimonio vivo, en continua evolución o a la idea de que el PCI no debe ser considerado producto, sino proceso. La "comunidad performativa", implicada en la patrimonialización incluiría a los vecinos de patios, los residentes en cualquier barrio de Córdoba, las asociaciones festivas y de vecinos, los empresarios del turismo -hosteleros y restauradores-, la administración local y regional, los expertos y académicos de la patrimonialización. Sin embargo, esta inclusividad abre la cuestión de si en la definición patrimonial deben tener todos la misma legitimidad y capacidad de decisión. En esta Fiesta de los Patios, por ejemplo, uno de los colectivos interesados son los turistas, lo que plantea el tema de si deben éstos tener la misma capacidad de decisión que los locales. Lo mismo puede plantearse, por ejemplo, de los touroperadores. ¿Acaso no son un grupo con intereses bien palpables en la promoción del destino? En un caso del PCI como el de la Fiesta de los Patios de Córdoba, donde ahora mismo uno de los principales problemas es la saturación turística y la masificación que está poniendo en peligro muchos de los elementos caracterizadores de este patrimonio, una definición laxa y no diagnosticada de "comunidad performativa" puede ser precisamente una forma de acabar con lo que se está pretendiendo proteger. 
Así, los dos conceptos fundamentales de comunidad o grupo que se están construyendo teóricamente, pueden llegar a ser opuestos en sus objetivos, intencionalidades y resultados. Pues como afirma Hertz, "Si la Convención [de 2003] buscaba garantizar, si no derechos, al menos reivindicaciones de grupos y poblaciones minoritarias, étnica e históricamente constituidos, el Grupo de Expertos define a las comunidades, grupos e individuos como redes, contingentes, colectivos basados en prácticas o performances (...). Al adoptar una concepción performativa de la base social del PCI, el Grupo de Expertos en esencia ha privado a estos actores sociales (relacionalmente constituidos) de las bases (estratégicamente reificadas) sobre las que afirmar sus reivindicaciones fundacionales contra los otros" (2015:35-36, la traducción es nuestra). Es decir, la propia flexibilidad conceptual del concepto de grupo hace que deje de poder ser usado como arma en diferentes reivindicaciones y, por lo tanto, produciendo efectos contrarios al empoderamiento que tienen que ver con procesos de desafección ydesapropiación del patrimonio.

\section{LAS LÓGICAS DE LA PARTIPACIÓN Y SUS POLARIDADES}

Señalábamos arriba que uno de los factores de éxito de la Convención 2003 es su componente democratizador. El giro participativo llega al patrimonio mundial no sin antes haber pasado y haberse ido extendiendo desde la década de 1970 en políticas públicas, planificación urbanística o programas de desarrollo(Cornwall, 2008: 269). Tampoco el debate sobre la necesidad de dar voz y participación a los colectivos puede decirse que sea una innovación absoluta de la Convención, desde las lógicas de la nueva museología ${ }^{12}$ (Rivière, 1993), hasta el cambio conceptual de patrimonio a patrimonialización (Sánchez-Carretero, 2012a, 2012b) inciden en este ámbito. El que la participación social en el ámbito cultural y patrimonial esté cada vez más presente, muestra una deriva de las nuevas lógicas de gobernanza a nivel internacional que sitúan la democratización de la gestión como un elemento fundamental de los actuales Estados; este proceso tiene defensores y también acumula progresivas críticas, muy a menudo asociadas a su instrumentalización o uso parcial e interesado.

En el ámbito patrimonial las lógicas de participación en los textos normativos aparecen ya hace décadas, por ejemplo en la Carta de Santiago de Chile de 1972 o en la Carta Internacional para la Gestión del Patrimonio Arqueológico de 1990; y en el contexto español algunos autores han venido analizando esta dimensión a través de diferentes trabajos que se centraban en la necesidad de tener presente la participación de los ciudadanos en el análisis de los procesos de patrimonialización (por ejemplo enAguilar,

12. El modelo de ecomuseos franceses de Rivière propone la musealización en el territorio como ámbito de participación social y política, al menos idealmente y da gran importancia a los modos de vida y formas de expresión de los diversos colectivos locales como elementos dignos de salvaguardia (Rivière, 1993). 
1999; Arrieta, 2009; Durán, 2007; Prats, 2003, 2005, 2012; Quintero, 2011). Sin embargo, hasta hace poco la relación entre participación y patrimonio en España estaba mayoritariamente organizada en torno a dos polaridades.

La primera de estas polaridades, estaría formada por la percepción -todavía mayoritariade expertos y técnicos de patrimonio que se adscriben a concepciones vinculadas al "discurso patrimonial autorizado" (Smith, 2006), que entienden que la población debe ser sensibilizada, educada, e incluso encuestada para evaluar resultados, al final de la cadena de valor del patrimonio (Criado-Boado, 1996; Criado-Boado y Barreiro, 2013) pero que no debe inmiscuirse en la identificación, diagnóstico o gestión directa de los bienes culturales. Este modelo, traducido a alguna de las escalas participativas (por ejemplo, la más clásica de Arnstein, 1969), respondería a modelos no-participativos en cuanto a que el valor de los bienes se considera objetivo y por ello legítimamente diagnosticado por expertos, cuando no pasa a formatos "tokenistas" o cosméticos, de participación sólo nominal o creando falsas apariencias participativas: informamos, divulgamos o consultamos, pero sin que los colectivos o grupos tengan derechos reconocidos de modificar ni los valores ni las formas de gestión del patrimonio.

Por otra parte, la segunda polaridad, estaría constituida por el creciente protagonismo, desde hace dos o tres década, de colectivos y grupos sensibilizados con diferentes bienes patrimoniales. Es lo que Ariño (2002) ha llamado "un nuevo actor del patrimonio" constituido por los movimientos sociales de reivindicación patrimonial en forma de "Salvemos", "Plataformas en defensa de", "Agrupaciones", etc. Diversos autores han analizado estos procesos en el contexto español (por ejemplo, Albert, 2005; Arrieta, 2009; Gómez Ferri, 2004; Hernández-Ramírez, 2005; Quintero, 2009; Santamarina, 2014). La red de actores que conforman estos movimientos patrimonialistas van a reivindicar su derecho a ser los protagonistas de la patrimonialización. Bien desde acciones de denuncia, reivindicación o erigidos en un nuevo interlocutor, asistimos a modelos de patrimonialización -aunque no sean los que más espacios o presupuestos ocupan en los catálogos oficiales- en los que se activan procesos de autogestión, aprendizaje colectivo, empoderamiento, en definitiva, en los que hay una fuerte implicación ciudadana (Hernández-Ramírez, 2003, 2005; Hernández y Ruíz, 2008 ).

Desde principios del siglo XXI nuevos textos y convenciones hacen valer la importancia de la participación en el diagnóstico y gestión patrimonial, por ejemplo: la Convención para la Salvaguarda del Patrimonio Cultural Inmaterial de la UNESCO (2003); la Convención sobre el Valor Patrimonial Cultural para la Sociedad del Consejo de Europa, firmada en Faro (2005); el Plan Nacional de Salvaguardia del Patrimonio Inmaterial del Ministerio de Educación Cultura y Deportes del Gobierno de España (2011); o el Manual de Gestión del Patrimonio Mundial Cultural de UNESCO, ICCROM, ICOMOS, UICN (2014). Esta profusión normativa en torno a la participación en patrimonio ha ido cambiando los 
discursos de políticos y técnicos (Sánchez-Carretero y Jiménez Esquinas, 2016) y ha dado cierto espacio a formatos, talleres y protocolos impulsados desde diferentes instancias administrativas. Así pues entre las dos polaridades antes mencionadas, de expertos que reniegan de la participación y movimientos autoorganizados, se expanden hoy programas y fórmulas para incentivar la sensibilización, la implicación y el compromiso de los ciudadanos con su patrimonio. Desde niveles locales o autonómicos se impulsa la participación en la elaboración de inventarios, se organizan foros de participación o se invita a asociaciones y otros grupos a seminarios o talleres mixtos dirigidos a "depositarios" del patrimonio y a técnicos que trabajan en patrimonio ${ }^{13}$.

Sin embargo, a menudo los programas de participación impulsados desde las instituciones son observados con suspicacia o desconfianza. Las experiencias de diferentes ciudadanos en procesos de participación, por ejemplo en los ámbitos de presupuestos municipales participativos, planes de desarrollo sostenibles dedicados a la conservación medioambiental o en planes de desarrollo rural, han tenido resultados muy desiguales y a menudo han sido frustrantes para los vecinos (Jiménez y García, 2016) ${ }^{14}$. Algunos de estos procesos en los ámbitos patrimoniales han adolecido de un carácter instrumental o de un uso "cosmético" de la participación (Coca, 2008;Coca y Escalera, 2014; Durán, 2007; Sánchez-Carretero, 2012b), sin que se hayan visto implementadas las propuestas desarrolladas.

Especialmente significativo, también por sus implicaciones políticas y por su carácter de modelo a seguir en los regímenes patrimoniales estatales, nos parece el modo en que se está implementando la Convención 2003 de la UNESCO. En teoría, los objetivos perseguidos por UNESCO dan prioridad a los derechos humanos, la sostenibilidad y una mayor justicia social, prioridades que pasan por dar protagonismo e involucrar a las "comunidades, grupos y en algunos casos individuos" considerados portadores del PCI. Pero, a pesar de la relevancia dada a los colectivos locales en la Convención de 2003, su protagonismo no es tal y muchos autores (Bendix et al., 2012; Hertz, 2015; Tauschek, 2015; Villaseñor y Zolla, 2012; Waterton y Smith, 2010) señalan que este "paradigma

13. Por ejemplo, las propuestas de Red de Agentes del Patrimonio Inmaterial de Andalucía o los Seminarios de Patrimonio Inmaterial organizados por el IAPH. Véase: http://www.juntadeandalucia. es/cultura/blog/patrimonio-inmaterial-y-participacion-social-el-atlas-del-patrimonio-inmaterial-deandalucia-y-los-seminarios-de-patrimonio-inmaterial-como-espacio-abierto-de-dialogo/

14. Jiménez y García (2016) citan entre las causas y procesos de frustración participativa: 1. La falta de continuidad de los procesos; 2 . la no implementación o deficiente puesta en práctica de las decisiones (lentitud, incumplimiento, modificación de acuerdos por parte de la administración); 3. el que sean procesos con capacidad de decisión en ámbitos muy limitados, no son ambiciosos o no tienen impacto; 4. la fatiga y aburrimiento, el verse sometidos a talleres y procedimientos considerados infantiles o que hacer perder mucho tiempo en relación a los resultados. 
participativo" no ha producido una mayor presencia de las comunidades locales en los procesos de toma de decisión en relación al patrimonio mundial y, en general, tampoco en cuanto a las selecciones patrimoniales regionales o estatales. Así mismo, también se ha detectado en estos trabajos que el efecto de empoderamiento para las "comunidades, grupos y en algunos casos individuos", que supuestamente traería consigo el giro participativo, dista mucho de haberse producido.

\section{CONCLUSIONES: IMPLICARSE, APROPIARSE, EMPODERARSE... LOS VERBOS DE LA PARTICIPACIÓN SOCIAL Y SUS CONJUGACIONES}

Los verbos de la participación tienen que ver con el ideal de la modernidad de democratizar la sociedad: empoderar, implicarse, apropiarse... En algunos casos la implicación de la población o de un sector, en la resolución de un problema concreto ha llevado a procesos de aprendizaje, mayor cohesión social, colaboración, etc. Sin embargo, los estudios realizados hasta ahora muestran la fractura social que se produce entre "comunidades, grupos y en algunos casos individuos" cuando se intentan implementar políticas patrimoniales participativas diseñadas rígidamente o sin contrastar en cuanto a su adecuación. En esta última sección del artículo, vamos a detallar algunas de las razones por las cuales los verbos de la participación no se conjugan sino que se quedan en su forma de infinitivo, que es la forma nominal que permite pasar de acción a ser sustantivo. Es decir, las razones por las que el propio paradigma participativo suele fosilizarse. Se detallan cuatro ejes que tienen que ver 1) con estructuras de poder y sistemas burocráticos, 2) significantes vacíos y expropiación de significados, 3) cuidados y afectos; y 4) el turismo y las economías del patrimonio. Así, algunas de las razones que hemos detectado por las que el "paradigma participativo" no consigue conjugar los verbos de la participación son:

1. Porque las comunidades y los colectivos locales están intermediadas por sistemas burocráticos y administrativos regionales y estatales que tienen mayor poder; además estos sistemas buscan actores fácilmente identificables y que no siempre representan adecuadamente a los colectivos y su complejidad. Véase, por ejemplo, la selección de asociaciones y colectivos representativos de la Dieta Mediterránea que en el caso español se concreta en asociaciones gastronómicas, de productores agroalimentarios, miembros de clubs UNESCO y actores académicos e institucionales. En esta selección, no quedan claros los criterios por los que se considera que unos actores son los más representativos respecto a una cultura alimentaria frente a otros. Otros casos y situaciones son aún más complicadas en tanto que implican una tecnocratización de la participación, seleccionando como interlocutores o "portadores" a ciertas 
asociaciones que representan a una parte del sentir y de las formas de interpretar las fiestas y tradiciones. Ejemplos de ello son los que se están produciendo en el caso de la Patum de Berga descrito por Noyes (2012) o en el caso de la Fiesta de los Patios de Córdoba. El problema no es que esas asociaciones no sean parte de la comunidad o del colectivo local, la cuestión es si ciertamente representan a todo ese colectivo, su diversidad, sus conflictos en la interpretación y usos del PCI. De nuevo cómo se defina la comunidad y el grupo, tal como exponíamos atrás, se vuelve fundamental.

2. Porque el modelo de patrimonialización obliga a un modelaje o adaptación al lenguaje y sistema del patrimonio, lo que puede llegar a suponer una expropiación de significados. Es decir, lleva a aceptar como incuestionables unas reglas del juego que responden a los formatos establecidos por el régimen patrimonial. La descripción y delimitación de valores de un saber, una técnica, una tradición o una fiesta, se traduce al lenguaje patrimonial, a lo que se considera más valorable en ámbitos internacionales, minimizando u ocultando, por ejemplo, elementos políticamente incorrectos -como la minusvaloración de las mujeres o ciertos elementos xenófobos- y ensalzando valores antes secundarios -como la puede ser la densidad histórica o los saberes relacionados con la sostenibilidad ambiental-. A veces este constreñimiento de valores puede llegar a usurpar los significados que los colectivos locales daban al bien, aunque éste así se transforma y se hace adecuado para otras audiencias y nuevos espectadores como la comunidad nacional e internacional o los turistas, por ejemplo. ¿Donde quedan aquí las voces de los "depositarios” de la tradición? Algunos autores señalan así la domesticación de las diferencias y la dominación simbólica que se produce en estos procesos de patrimonialización (Villaseñor y Zolla, 2012; Kuutma, 2012 citado en Tauschek 2015).

3. Porque al mismo tiempo se crean nuevas necesidades y se demandan una serie de actividades, trabajos, e inversiones de mantenimiento del saber -o del ritual, o de la actividad- que recaen sobre los colectivos de un modo desigual. Las prácticas, que antes eran de uso y gestión local, han pasado a ser patrimonio del común -de los habitantes de una región, de una nación, de la comunidad internacional-pero las tareas de realización, celebración, sostenimiento, los cuidados, las emociones invertidas (Jiménez-Esquinas, 2014) son repartidas de modo jerárquico y no equivalente a la toma de decisiones. Otras veces lo que se produce es un distanciamiento absoluto entre las expresiones culturales que se patrimonializan y la dimensión social; las condiciones de vida real, las situaciones de sufrimiento, dificultades, miserias, las corporalidades, se ocultan o se niegan (Fernández Juárez, 2013; Jiménez-Esquinas, 2014).

4. Porque la selección de qué elementos se propongan como PCI mundial o en los catálogos nacionales suelen servir a los propósitos económicos o de construcción identitaria hegemónica que interesa a los gobiernos y aparatos del Estado. A pesar 
de concebirse este patrimonio en función de su representatividad, es frecuente que se seleccionen por parte de los Estados y se proclamen como parte del PCI mundial las prácticas más singulares o espectaculares, fácilmente integrables en lógicas turísticas y de consumo global. Otra opción reiterada es la selección de elementos que sirven para construir una imagen deseada de la nación, ya sea integrando colectivos étnicos diferenciados y convirtiéndolos en la amalgama constructora del Estado-Nación, ya sea seleccionando ciertos elementos connotados en el debate Estatal y pretendiendo erigirse en representativos de todo el conjunto ${ }^{15}$.

El giro hacia el paradigma de la participación en patrimonio necesita urgentemente el desarrollo de mecanismos de diseño, análisis y evaluación. Corremos el riesgo de que la "tiranía de la participación" (Cooke y Kotari, 2001) burocratice estos procedimientos y, en algunos casos, invierta los resultados esperados, por ejemplo respecto a implicación y empoderamiento de comunidades, grupos, etc. Sería necesario conocer qué tipos de procesos de participación se están poniendo en marcha en distintos ámbitos, qué características tienen, y, sobre todo, con qué recursos -humanos, técnicos, presupuestarios- cuentan. Ya que, entre otras cosas, la participación puede implicar el riesgo de una lógica del "coste 0 " o de diluir competencias y responsabilidades en esa "ciudadanía participativa" que siente como propio su patrimonio.

Una de las consecuencias del paradigma participativo está relacionada con las contradicciones implícitas que existen el definir quién participa, quién se erige o es erigido como la "comunidad, grupo y en algunos casos los individuos". Además de esta consecuencia, el imperativo de incluir procesos participativos tiene una segunda consecuencia, que es la necesidad de realizar análisis y diagnósticos en profundidad sobre los agentes patrimoniales y los procesos de participación. Estas tareas implican promover un tipo de conocimiento técnico muy concreto: "los especialistas en participación". Así, una nueva demanda de técnicos se introduce en el régimen patrimonial para los que la antropología está muy bien equipada, pero que choca con las limitaciones de tiempo y, sobre todo, las limitaciones de financiación que la gestión patrimonial suele llevar aparejada. Nos parece importante señalar el riesgo implícito que puede llevar a que las personas especialistas en participación se transformen en facilitadoras de un patrimonio participativo 2.0 a coste cero.

Los análisis sobre procesos participativos y sus efectos vinculados a otros campos, como medioambiente, políticas públicas o cooperación y desarrollo, suelen sustentarse, de una forma más o menos explícita, en principios morales sobre los efectos "buenos" o "malos" que se consiguen a través de los procesos participativos, estableciéndose gradientes de

15. El interés del Gobierno español por gestionar directamente el Patrimonio Inmaterial ha quedado de manifiesto en el desarrollo legislativo que viene haciendo, sin contemplar que las del Patrimonio son competencias transferidas de pleno derecho a las Comunidades Autónomas. Esta situación ha sido analizada con claridad por Gema Carrera (2016). 
formas "malas" hacia formas "buenas" de participación (Cornwall, 2008: 271). Aquí planteamos la importancia de analizar los propios términos sobre los que se construye la participación. Parafraseando a Hafstein, la fuerza magnética de lo patrimonial hace que se pase fácilmente a criticarlo usando los propios términos sobre los que se asienta el régimen patrimonial, en vez de analizar dichos términos (Hafstein, 2012:501). En este artículo hemos puesto en cuestión los verbos que se vinculan con la participación, sobre los que se asienta el giro participativo en patrimonio, así como la ausencia de sus formas verbales conjugadas. Entender los términos sobre los que se asienta el régimen patrimonial y el papel de los procesos participativos en patrimonio, pasa por comprender qué procesos y fórmulas de participación se están poniendo en marcha desde las administraciones de gestión del patrimonio; comparar los distintos regímenes patrimoniales en el Estado y Autonomías y su relación con las actuaciones de la UNESCO.

También pasa por comprender que la lógica del "coste 0" que está detrás de muchos procesos participativos implica una violencia estructural del régimen patrimonial que, probablemente, desencadenará una desafección por el patrimonio. Este es uno de los efectos de las instrumentalizaciones de la participación que se puede y debe minimizar trabajando con fórmulas de diagnóstico inicial de colectivos que incluyan a actantes (humanos y no humanos como normativas, procedimientos, leyes o planes directores) y que partan del conocimiento de lo que "hace" la participación en el régimen patrimonial. 


\section{REFERENCIAS BIBLIOGRÁFICAS}

Adell, Nicolas; Bendix, Regina; Bortolotto, Chiara y Tauschek, Markus (2015) Between Imagined Communities and Communities of Practice: Participation, Territory and the Making of Heritage. Göetingen: Universitätsverlag Göttingen.

Adell, Nicolas (2015) "Polyphony vs. Monograph: The Problem of Participation in a French ICH Dossier". En Nicolas Adell, Regina Bendix, Chiara Bortolotto y Markus Tauschek (eds.)Between Imagined Communities and Communities of Practice:Participation, Territory and the Making of Heritage. Göttingen: Universitätsverlag Göttingen, pp. 237248.

Agudo Torrico, Juan (1999) "Cultura, patrimonio etnológico e identidad" . Boletín del Instituto Andaluz del Patrimonio Histórico 29 pp. 36-45.

Aguilar, Encarnación (ed.) (1999) Patrimonio Etnológico. Nuevas Perspectivas de Estudio. Granada: Diputación de Granada.

Aguilar, Encarnación y Santiago Amaya (2007) "El patrimonio cultural como activo del desarrollo rural". En J. Sanz (ed.) El futuro del mundo rural. Sostenibilidad, innovación y puesta en valor de los recursos locales. Madrid: Síntesis, pp. 103-124.

Albert, Jean Pierre (2003) "Patrimonio y etnología en el sur de Francia”.En González Alcantud (ed.) Patrimonio y pluralidad. Nuevas direcciones en antropología patrimonial. Granada: Centro de Investigaciones Etnológicas Angel Ganivet, Diputación Provincial de Granada, pp. 247-270.

Albert Rodrigo, María (2005) "El patrimonio cultural y la sociedad civil". En Gil Manuel Hernàndez i Martí, Beatriz; Santamarina Campos y Albert Moncusí Ferré (coords.) La memoria construida. Patrimonio cultural y modernidad.Valencia: Tirant lo Blanch, pp. 193-224.

Alonso González, Pablo (2013) The Heritage Machine: A Heritage Ethnography in Maragatería (Spain).Tesis doctoral: Universidad de León.

Alsayyad, Nezar (2001) Consuming tradition, manufacturing heritage: global norms and urban forms in the age of tourism. London: Routledge.

Ariño, Antonio (2002) “La expansión del patrimonio cultural". Revista de Occidente 250, pp. 129-150.

Arnstein, Sherry R. (1969) "Ladder of Citizen Participation".JAIP (Journal of the American Institute of Planners) 35(4), pp. 216-224.

Arrieta, Iñaki (2009) "Comunidades, científicos y especialistas en los proyectos patrimoniales y museísticos: de «arriba-abajo», de «abajo-arriba»". En Iñaki Arrieta (ed.)Activaciones patrimoniales e iniciativas museísticas: ¿por quién?, ¿para qué?.Bilbao: Servicio de Publicaciones de la Universidad del País Vasco, pp. 11-19. 
Arizpe, Lourdes (2004) "El patrimonio inmaterial, la diversidad y la coherencia".Museum International 221, pp. 133-140.

Baron, Roberty Spitzer, Nick (2007 [1992]) Public Folklore. Jackson: University Press of Mississippi.

Bendix, Regina (2011) "Héritage et patrimoine: de leurs proximités sémantiques et de leurs implications".En Chiara Bortolotto (ed.) Le patrimoine culturel immatériel : enjeux d'une nouvelle catégorie.Paris: Éditions de la Maison des sciences de l'homme, pp. 99-121. Bendix, Regina; Eggert, Aditya y Peselmann, Arnika (2012) Heritage Regimes and the State. Göttingen: Universitätsverlag Göttingen.

Blake, Janet (2009) “UNESCO's 2003 convention on intangible cultural heritage: the implications of community involvement in 'safeguarding"'. En Smith, L. y Akagawa, N. (eds.) Intangible heritage, London, Routledge, pp. 45-73.

Bortolotto, Chiara (2011) "Le trouble du patrimoine culturel immatériel”. En Chiara Bortolotto (ed.) Le patrimoine culturel immatériel: enjeux d'une nouvelle catégorie.Paris: Éditions de la Maison des sciences de l'homme, pp. 21-46.

Bourdieu, Pierre(1998[1978]) La distinción. Criterio y bases sociales del gusto. Madrid: Taurus.

Bruman, Christoph (2015) "Community as a Myth and Reality in the UNESCO World Heritage Convention". En N. Adell, R. Bendix, C. Bortolotto y M. Tauschek (eds.)Between Imagined Communities and Communities of Practice:Participation, Territory and the Making of Heritage.Göttingen: Universitätsverlag Göttingen, pp. 273-289.

Carrera, Gema (2005) "La evolución del patrimonio (inter) cultural: políticas culturales para la diversidad". En G. Dietz y G. Carrera (eds.) Patrimonio cultural, multiculturalismo y gestión de la diversidad. Sevilla: Consejería de Cultura, pp. 14-29.

Carrera, Gema (2016) Propuesta metodológica para la documentación y gestión del patrimonio cultural inmaterial como estrategia de desarrollo social y territorial. Tesis doctoral, Universidad de Sevilla.

Collomb, Gérard (1999) "Ethnicité, nation, musée en situation post-coloniale".Ethnologie Française, XXXIX(3),pp. 333-336.

Cornwall, Andrea (2008) "Unpacking 'Participation': models, meanings and practices". Community Development Journal43(3), pp. 269-283.

Cortés Vázquez, José Antonio; Jiménez-Esquinas, Guadalupe y Sánchez-Carretero, Cristina (2017). "Heritage and Social Participation: critical approaches and new comparative strategies". Anthropology Today33(1), pp. 15-19. 
Criado-Boado, Felipe(1996) "Hacia un modelo integrado de investigación y gestión del Patrimonio Histórico: la cadena interpretativa como propuesta”. PH Boletín16, pp. 73-78. Criado-Boado, Felipe y Barreiro, David (2013) "El patrimonio era otra cosa". Estudios atacameños45, pp. 5-18.

Douglas, Mary (1998) Estilos de pensar. Barcelona: Gedisa.

Durán Salado, Isabel (2007) Participación y percepción social en la gestión de conjuntos patrimoniales. Sevilla: IAPH.

Foucault, Michel (1977) "The political function of the intellectual". Radical Philosophy, 17, pp. 12-14.

García Canclini, Néstor(1999) "Los usos sociales del Patrimonio Cultural". EnE. Aguilar (coord.) Patrimonio etnológico. Nuevas perspectivas de estudio. Sevilla: Instituto Andaluz de patrimonio Histórico, pp. 16-33.

García García, José Luis (1998) "De la cultura como patrimonio al patrimonio cultural". Política y Sociedad27, pp. 9-20.

Gómez Ferri, Xavier (2004) "Del patrimonio a la identidad. La sociedad civil como activadora patrimonial en la ciudad de Valencia”.Gazeta de Antropología 20, pp. 9-20 http://hdl.handle.net/10481/7260[Consultado el 10 de octubre de 2016].

Guillaume, Marc (1990) “Invention et stratégies du patrimoine”. En H. P. Jeudy (dir.) Patrimoines en Folie.París: Maison des Sciences de l'Homme, pp. 12-20.

Hafstein, Valdimar (2009) "Intangible Heritage as a List: from Masterpieces to the Convention for the Safeguarding of Intangible Cultural Heritage". En L.Smith y AkagawaN.(eds.)Intangible Heritage. London: Routledge, pp. 93-111.

Hafstein, Valdimar (2011) "Célébrer les différences, renforcer la conformité". En Chiara Bortolotto (ed.) Le patrimoine culturel immatériel: enjeux d'une nouvelle catégorie.Paris: Éditions de la Maison des sciences de l'homme, pp. 75-97.

Hafstein, Valdimar (2012) “Cultural Heritage”. En R. Bendix y G. Hasan-Rokem (eds.)A Companion to Folklore. Oxford: Wiley Blackwell, pp. 500-519.

Heinich, Nathalie (2009) La fabrique du patrimoine. «De la cathédrale à la petite cuillère». Paris: Maison des Sciences de l'Homme.

Hernández-Ramírez, Javier (2003) "La construcción social del patrimonio: selección, catalogación e iniciativas para su protección. El caso del Palacio del Pumarejo". En V. Quintero y E. Hernández (coords.) Antropología y Patrimonio: investigación, documentación e intervención. Granada: Junta de Andalucía, pp. 84-95. 
Hernández-Ramírez, Javier (2005) "De resto arqueológico a patrimonio cultural. El movimiento patrimonialista y la activación de testimonios del pasado". Boletín GC: Gestión Cultural, 11. http://www.gestioncultural.org/ficheros/1 1316765099 bgc11Hernandez.pdf[Consultado el 10 de octubre de 2016].

Hernández Ramírez, M. y Ruiz Ballesteros, E. (2008) "El patrimonio como proceso social. Intervención, desarrollo y consumo del patrimonio minero andaluz". En Arrieta, I. (coord.) Participación ciudadana, patrimonio cultural y museos: entre la teoría y la praxis. Bilbao: Servicio Editorial de la Universidad del País Vasco, pp.129-148.

Hertz, Ellen (2015) "Bottoms, Genuine and Spurious". En N. Adell, R. Bendix, C. Bortolotto y M. Tauschek (eds.)Between Imagined Communities and Communities of Practice:Participation, Territory and the Making of Heritage. Göttingen: Universitätsverlag Göttingen, pp. 25- 57.

Hymes, Dell (2000 [1975]) “La naturaleza del folklore y el mito del sol”. En C. SánchezCarretero y D. Noyes (eds.) Performance, arte verbal y comunicación. Nuevas perspectivas en los estudios de folklore y cultura popular en USA. Oiartzun: Sendoa, pp. 55-73. Jeudy, Henry-Pierre (2001) La machinerie patrimoniale. Paris: Sens et Tonka.

Jiménez-Esquinas, Guadalupe (2014)Crafting and Affective Landscapes: heritagization processes of textile crafts and landscapes in Costa da Morte (Galicia, Spain).Institute of Heritage Sciences (Incipit-CSIC) and University of the Basque Country (EHU), Tesis doctoral inscrita.

Jiménez Sánchez, Manuel y García Spín,Patricia (2016) "Newsletter: Hoy hablamos demalas noticias: La frustración participativa". En Cherry-picking: the results of participatory processes / Los resultados de los procesos participativos: políticas públicas y relación administración-sociedad. IESA (CSIC)https://cherrypickingproject.wordpress. com/ [Consultado el 10 de octubre de 2016].

Kirshenblatt-Gimblett, Barbara (2004) "Intangible Heritage as Metacultural Production". Museum International 56(1-2), pp. 52-65.

Kurin, Richard (2004) "Safeguarding Intangible Cultural Heritage in the 2003 UNESCO Convention: A Critical Appraisal”.Museum International 56(1-2), pp. 66-77.

Matsuura, Koichiro (2000) “Está creando la globalización de la economía valores para una nueva civilización?”Perspectivas. Revista trimestral de Educación Comparada, OIE/ UNESCO XXX(4).

Matsuura, Koichiro (2004) “Preface”.Museum International 56(1-2), pp. 4-5.

Noyes, Dorothy (2012) “The Social Base of Folklore". En R. Bendix y G. Hasan-Rokem (eds.)A Companion to Folklore. Oxford: Wiley Blackwell, pp. 13-39. 
Pazos Cárdenas, Mateo (2016) "Las industrias culturales afropacíficas. Encrucijadas del multiculturalismo en la ciudad de Cali, Colombia”.Antípoda: Revista de Antropología y Arqueología 24, pp. 75-90.

Pérez Galán, Beatriz (2008) "El Patrimonio Inmaterial en proyectos de desarrollo territorial en Comunidades Indígenas de Los Andes Peruanos”.Revista Electrónica de Patrimonio Histórico 3, pp. 1-29.

Pérez Galán, Beatriz (2011) "Los usos de la cultura en el discurso legislativo sobre patrimonio cultural en España. Una lectura antropológica sobre las figuras legales de protección”. Revista Experimental de Antropología 11, pp. 11-30http://revistaselectronicas. ujaen.es/index.php/rae/article/view/1887/1639 [Consultado el 12 de octubre de 2016].

Prats, Llorenç (2003) “Patrimonio + turismo = ¿desarrollo?”.Pasos, Revista de Turismo y Patrimonio Cultural 1(2), pp. 127-136, www.pasosonline.org

Prats, Llorenç (2005) “Concepto y gestión del patrimonio local”.Cuadernos de Antropología Social 21, pp. 17-35

Quintero Morón, Victoria (2005) "El patrimonio intangible como instrumento para la diversidad cultural ¿una alternativa posible”. EnG. Dietzy G. Carrera(eds.) Patrimonio cultural, multiculturalismo y gestión de la diversidad. Sevilla: Consejería de Cultura, pp. 68-83.

Quintero Morón, Victoria (2011) “El patrimonio pertenece a todos. De la universalidad a la identidad, ¿cuál es el lugar de la participación social?”.En Inaki Arrieta Urtizberea (ed.) Legitimaciones sociales de las políticas patrimoniales y museísticas. Abadiño: Universidad del País Vasco, pp. 45-78.

Rosas Mantecón, Ana (1999) "La participación social en las nuevas políticas para el Patrimonio Cultural".En Patrimonio etnológico. Nuevas perspectivas de estudio. Granada: Instituto Andaluz de Patrimonio Histórico, Consejería de Cultura. Junta de Andalucía y Comares, pp. 34-51.

Rioja, Concha (1996) "Reflexiones en torno a la cultura inmaterial y su gestión patrimonial en la Comunidad Autónoma Andaluza”. PH Boletín del Instituto Andaluz del Patrimonio Histórico, 16, p. 79-16.

Sánchez-Carretero, Cristina (2005) "Sobre el patrimonio inmaterial de la humanidad y la lucha por visibilizar «lo africano» en la República Dominicana”. En X.C. Sierra y X. Pereiro (eds.)Patrimonio cultural: politizaciones y mercantilizaciones. Sevilla: Fundación El Monte, pp. 147-163. 
Sánchez-Carretero, Cristina (2012a) "Hacia una antropología del conflicto aplicada al patrimonio". En B. Santamarina Campos(ed.) Geopoliticas patrimoniales. De culturas, naturalezas e inmaterialidades. Una mirada etnográfica. Valencia: Germania, 2012, pp. 195-210

Sánchez-Carretero, Cristina (2012b) "Heritage Regimes and the Camino de Santiago: Gaps and Logics."En R. Bendix, A. Eggert,y A. Peselmann (eds.) Heritage Regimes and the State. Göttingen: Universitätsverlag Göttingen, pp. 141- 155.

Santamarina Campos, Beatriz (2009) "De parques y naturalezas. Enunciados, cimientos y dispositivos". Revista de Dialectología y Tradiciones PopularesLXIV(1), pp. 297-324.

Santamarina Campos, Beatriz (2013) "Los mapas geopolíticos de la Unesco: entre la distinción y la diferencia están las asimetrías. El éxito (exótico) del patrimonio inmaterial”. Revista de Antropología Social22, pp. 263-286.

Santana, Agustín (2002) "Mirar y leer: autenticidad y patrimonio cultural para el consumo turístico". En VI Encontro Nacional de Turismo com base local. Octubre. Campo Grande.

Segalen, Martine (2003) "Cuestiones de identidad y alteridad. La experiencia francesa del patrimonio". En González Alcantud (ed.) Patrimonio y pluralidad. Nuevas direcciones en antropología patrimonial. Granada: Centro de Investigaciones Etnológicas Angel Ganivet, Diputación Provincial de Granada, pp. 41-62.

Smith, Laurajane (2006) Uses of Heritage. London: Routledge.

Tauschek, Markus (2015) "Imaginations, Constructions and Constraints: Some Concluding Remarks on Heritage, Community and Participation". En N. Adell, R. Bendix; C. Bortolotto y M. Tauschek (eds.)Between Imagined Communities and Communities of Practice:Participation, Territory and the Making of Heritage. Göttingen: Universitätsverlag Göttingen, pp. 291-306.

Tunbridge, J. y G. Ashworth (1996) Dissonant Heritage: The Management of the Past as a Resource in Conflict. New York: John Wiley \& Sons.

UNESCO (2003) Convención para la Salvaguardia del Patrimonio Cultural Inmaterial., París, 17 de octubre de 2003.

Villaseñor, Isabel y Zolla, E. (2012) "Del Patrimonio Cultural Inmaterial o la patrimonialización de la cultura, Cultura y representaciones sociales". Revista del Instituto de Investigaciones Sociales6(12), pp. 75-101.

http://www.revistas.unam.mx/index.php/crs/article/view/30475) [Consultado El 4 de octubre de 2016].

Waterton, Emma y Smith, Laurajane (2010) "The recognition and misrecognition of community heritage".International Journal of Heritage Studies16,(1-2), pp. 4-15. 